\title{
CURRENT SCALING PRACTICES AND DEVELOPMENTS IN BRITISH COLUMBIA
}

\author{
By A. R. FRASER ${ }^{1}$ and C.J. HIGHSTED 2
}

\section{INTRODUCTION}

The assignment given by the Chairman of the Forest Mensuration Committee is to "cover current scaling practices and indicate improvements that will be required in light of current developments in harvesting methods" in British Columbia.

Scaling practices throughout North America have been dictated by numerous local customs, policies and operational factors. Since only a few well-known measurement fundamentals are involved, details are seldom of interest beyond the regional level. British Columbia scaling practices are no exception; hence current practices are covered in a general way with brief comments on local purposes and error objectives and a few slides to illustrate the physical aspect of scaling practices in British Columbia. Such coverage should imply a host of details to a group of foresters.

Improvements underway are also of a local nature. However, some of the longer term problems may be of a wider interest pattern. Certainly, the solutions must draw on much "outside" research and development work.

\section{Local Purposes}

The so-called Forest Service or Government scale is the general base for discussion of scaling practices in British Columbia. Originally the sole, and still the primary, purpose of this scale is to provide a basis for the Crown to Industry transaction involving stumpage and royalty payments. A second purpose, since 1952, has been as part of the allowable cut control required in forest units managed within concepts of sustained yield. This second purpose is also of interest to both the Crown and Industry who are both concerned with quotas or cutting budgets. Other Crown uses of the scales are of a more general statistical record nature.

The Forest Service scale is used by Industry for a wide variety of "buyer-only" interests. In most instances, it is the best scale ever made on the logs and it is adequate for many present day production control purposes, for subsequent buying, selling or trading of logs, or for subcontract payments. Therefore, in using the Government scaling practice as a base for indicating current practices in British Columbia much scaling for industry purposes is automatically included. However, Industry does scale privately for other reasons. In general these are less elaborate scales, tree counts and bush scales, for uses before the Government scale is made. A notable exception is the much more elaborate 'grade' scale, generally made only on those

\footnotetext{
${ }^{1}$ Forester, Research Division, British Columbia Forest Service, Victoria, B.C.

${ }^{3}$ Forester, Management Division, British Columbia Forest Service, Victoria, B.C.

This paper was read and illustrated with colour slides by J. S. Stokes at the 1965 Aanual Meering of the C.I.F.
} 
higher value Coast rafts which run heavy to peelers. 'Royalty Act' grades, based on lumber cutout relationships of earlier days are just too outmoded for use in a peeler transaction.

\section{ERror OBJEctives AND COSTS}

The longest standing measurement objective of Forest Service scales has been that two competent scalers should be able to apply the accepted scaling procedures to yield independent volume estimates of a given run of logs within $3 \%$ of each other. Scaler training and check scale details are geared to this objective and it is generally achievable, except where much judgment must enter small lots of defective material. In cases of dispute over a scale the same criterion is applied. The first scale stands if a rescale is within $3 \%$ and the buyer pays for the rescale. If more than $3 \%$ shows, the seller pays. In such cases mutual agreement is sought, usually by appraisal of volume and value errors in strata breakdowns, or further rescales are pursued. If anyone wants a definition of 'true' scale in B.C., this highly workable procedure is as close as we get.

This scaler repeatability criterion covers many measurement and judgment errors. But it could not acknowledge the growing inadequacies of the f.b.m. B.C. Log Rule as the basic unit of measure. This audience needs no evidence on how an empirical rule designed for log sizes and lumber cutting practices of 1894 becomes outmoded. There are some 50 rules just like it scattered around North America. Where scales are made in f.b.m., outmoded 'overrun' concepts and possibly grossly misleading estimates of volume must still be kept in mind in addition to application errors. Orchard (1952) discussed the local problem in detail. Subsequently, Government scaling in B.C. has been in cubic feet (Smalian application). While not 'perfect', the errors associated with application of this unit are nominal and of a more random nature, difficult to take intentional advantage of in buyer-seller propositions.

Normally, all logs are scaled and sampling errors are not a consideration. However, during the past 5 years, there has been a trend towards solving difficult operating situations by sample scaling. To date sampling has been applied only where log ownerships are not mixed and where log grading is not done, i.e. where species are the only important strata. Truckloads of small wood or of lower value salvage and pulpwood bundles have received most attention so far. Sample scale procedures in current use are:

(a) Total piece count $x$ average volume per piece.

(b) Total weight $x$ average volume per pound.

In all cases, the volume or volume ratio is determined by sampling. Standard statistical techniques are used in determining intensity and sampling error. Only unrestricted random or simple species stratification propositions have been entertained to date. Moreover, in the present development period, sampling error objectives are established at a constant $\pm 1 \%$ (p.05).

Detailed field measurement and office procedures, compatible with these error ojectives, follow more or less standard textbooks formats. One recording topic is notable. On the Coast, net log dimensions (after deduction for defect) are recorded and the volumes are computed and summarized by a specialized office staff. In the Interior, volumes are recorded directly by the scalers. 
On costs, Coast scaling is done by official scalers employed by the Forest Service, with costs charged to the Industry. Current costs, including data processing, are:

1. $24 \mathrm{c}$ per $M$ f.b.m.

2. $18 \mathrm{c}$ per $\mathrm{C}$ c.f.

3. 7c per $M$ f.b.m.

The first cost is charged for scales made in board measure only, acceptable for timber sold under certain old contract conditions, and is applicable to about $20 \%$ of the total cut.

The second cost is charged for modern Crown-Industry stumpage transations and is applicable to about $80 \%$ of the total cut.

The third cost is made for what is called a dual scale, being a secondary compilation from the cubic measurements and often required by the Industry for their interests only. About $70 \%$ of the total cut is compiled "dual".

Interior scaling is done by scalers licensed by the Government, but employed directly by the Industry (except sample scaling which is done by "official" scalers). Current costs range from $20 \mathrm{c}$ to $90 \mathrm{c}$ per C c.f. Board measure scaling is confined to small lots of privately-owned timber and costs are not available. The dual scale practice of the Coast is seldom used in the Interior.

Scales in other measures, such as cords, lineal feet, pieces, etc. are restricted to minor products.

\section{Physical Mediums}

Almost all Coast timber, currently about $50 \%$ of the B.C. cut, eventually reaches a flat raft stage in sheltered waters where the scaling activity takes place. Such a raft may contain anywhere from a few thousand to 30 thousand cubic feet. Normally several species, log grades, and timber marks (areas or contracts of origin) are mixed in a single raft. Such arrays of strata, plus multipurpose interests in each, preclude any attempt at sampling and it is normal practice to measure each log. Changes in harvesting practices, which have taken the Coast Industry from oxen to perhaps the most highly mechanized and integrated in the world, have had almost no effect on physical problems of scaling.

The other $50 \%$ of the annual cut, mostly in the Interior region of B.C., is scaled by dry-land procedures at some pre-designated point between bush and mill. In general the timber is smaller than on the coast. Here improvements in mechanizing logging, closer utilization and reduction of small bush mill in favour of larger mills in towns and villages has created 'hot' scaling situations. Many small logs, often in truckload batches pass the scaling points faster. Sample scaling is used increasingly.

\section{IMPROVEMENTS UNDERWAY}

In considering improvements, the kind presently being tackled assume no change in purpose of scaling, measurement objectives, nor in the GovernmentIndustry setup under which major scaling activity is conducted. Improvements underway are primarily to meet changing local operating situations. 
For example, day to day problems occur frequently in dry land scaling since the flow of logs is usually 'hot' from bush to jack ladder or rollway. A few new trucks, a new fast feed headsaw cutting small logs, or bundles of smallwood and salvage are likely to leave an individual scaler waving his stick at the logs rather than scaling them. Here the attack underway is to attempt the standard sampling procedures mentioned previously. Currently about $12 \%$ of the annual cut of the Interior is sample scaled. Expansion of this activity is anticipated and, given experience, improvements might include more sophisticated procedures and objectives, e.g., multistage sampling, multiple regression estimators by purposive selection, optimum allocation to profit or cost rather than constant error objectives, and so forth. Sample scaling is being developed on a local need basis. Industrial foresters facing particular operating situations propose a sampling scheme. Details and trial runs are completed in cooperation with the Forest Service. So far, rejection of proposals has usually been associated with too many strata of individual interest or too much variation from sound-defective material mixtures.

Coast scaling is less subject to the numerous changes in harvesting techniques that are occurring. The rafts have always provided a point of scaling where the production flow is normally halted and naturally spread for scaling until both buyer and seller are satisfied. The day to day pressures here are to speedup the scaling activity. Some streamlining of scalers' records and a switch from mechanical tabulation to electronic data processing are improvements underway. Industry are also constantly improving their sorting and booming grounds-among other things introducing more uniformity in individual rafts.

To our knowledge local formal research on topics related to scaling is limited to one project by the Federal Forest Products Laboratory-relating lumber cutout log grades to tree quality for some Interior species.

\section{Longer Term Problems}

Improvements generally follow some such motivating force as demands for new information, a change in purposes or use of scales, a change in law, or availability of new methods or measuring devices. Suggestions of longer term problems is therefore prognosis on motivating forces, fraught with the usual difficulties.

Government demands related to scaling are not likely to grow. Rather changes conducive to simplification of scaling might be anticipated. Thus, legal changes modernizing an old and complex tenure system, or eliminating outmoded Royalty Act grades would simplify strata of interest and hence scaling practice. Cruise rather than scale is often proposed as a base for Government-Industry relationships. However, other than noting that such possibilities might tend to simplify scaling for Government purposes, it would not have much effect on other needs for scales.

Prognosis of changes in Industry which may motivate different scaling practices are easier-leave it to the experts. Loggers, woods managers, management consultants and vice-presidents of the Industry have spelled them out 
quite clearly for the Pacific States, including B.C. (See several articles and discussion, Proceedings Pacific Logging Congress 1961, '62, '63 and particularly '64).

Summarizing only progressive viewpoints, the demand is for cubic foot scaling and better log-grading systems. Production control, in the sense of ensuring an approximate (f.b.m.) quantity of material of present log grades at the bottom of various jack ladders, is not sufficient for modern purposes. Quality control, if related solely to stages of manufacture beyond the top of the jack ladder, also fails to meet modern needs. The demand is for better control from stump to product-optimum allocation of raw material. Of course, more sophisticated controls will not work without reliable information-hence a demand for more useful scales is being voiced. Cubic log contents or some better fibre content measure and a more flexible grading system are vital needs discussed in Congress Proceedings. Solutions, impractical in the pre-computer age, are being suggested-gross, net and defect character measures of each $\log$-with intention of computing values to more than one end purpose for each $\log$ as part of the allocation decision.

Naturally such information must be available quickly, implying automation of scaling practice. Here, Grosenbaugh (1959) has provided the prognosis of means to ends for quantification in general-much of which could apply to scaling practice, e.g. Gross volume measures-photoelectrically; species identification-by chromatography, internal qualities-by sonics, and so forth.

The more integrated B.C. industries already have several of the prerequisites for better control. Most are computer oriented. All have been exposed to cubic scaling for 12 years (some using it for all purposes). Elaborate sorting grounds provide natural scaling gaps far enough from the jack ladder gap to be useful for overall control. Among the lacks are useful grading systems, basic quantity-quality relationships from tree to log and from log to product, fast scaling, and experience with operational tools such as system analysis and linear programming.

If, as, and when Industry translates this 'voiced' need for better control into an 'action' program, there should be no shortage of technical problems.

Many of these problems will be directly related to scaling practice, or otherwise within the working sphere of foresters with mathematical aptitudes.

\section{Cited}

GROSENBAUGH, L. R. 1959. Quantification and Estimation in Future Forest Management, Proc. Soc. Amer. Foresters.

ORCHARD, C D. 1952. Cubic Scale in British Columbia. Forest Topics No. 4, B.C. Forest Service.

PACIFIC LOGGING CONGRESS 1961-64. Proceedings in Loggers Handbooks, Volumes XXI to XXIV. 